\title{
Platelet-Rich Plasma Injection Therapy for Refractory Coccydynia: A Case Series
}

Fergie-Ross Montero-Cruz, DO${ }^{1}$, and Steve M. Aydin, DO²

Background: Coccydynia is pain in the coccyx region. The most common cause of coccydynia is trauma, either from a direct axial force such as during a fall onto the coccyx or from cumulative trauma as a result of poor sitting mechanics. Risk factors include obesity, female gender and rapid weight loss. The anatomy of the region is not well vascularized, and consists of the coccyx bones, and supporting ligaments and tendons. For a majority of patients, conservative management may be successful but for the remainder of patients, pain relief may only be transient and pain can become debilitating. What has yet to be fully explored is the possible beneficial effect of local injection of platelet-rich plasma (PRP) for the treatment of refractory coccydynia. PRP injections have been successful in treating a variety of chronic tendon, ligament and bone injuries by inducing an inflammatory response to promote or re-initiate healing. In addition, local injections of PRP to the coccyx would be a less invasive option than surgery for patients who have exhausted all other treatment options and continue to struggle with the debilitating effects of coccydynia.

Objectives: To demonstrate the important role of PRP therapy in treating coccydynia refractory to traditional conservative management.

Study Design: Retrospective case series.

Methods: Three patients with coccygeal pain for greater than 6 months who presented to an outpatient pain management office in New York from 2014 until 2016, and failed conventional treatments. When the pain was deemed refractory for the patient, offering for PRP was done. This was presented with the option to repeat once again at the 6-8 week mark if needed. All patients underwent PRP with fluoroscopic injection, and the use of the same PRP commercially available system; Magellan-Arteriocyte. The patients were evaluated with a numerical rating scale, and percentage of pain relief at 6-8 week post-injection, 6 months post-injection, 12 months post-injection, and 24 months post-injection was recorded. Percentage of pain relief was then calculated.

Results: Overall the patient series demonstrated significant improvement in numeric pain scale, as well as percentage of pain reduction. At the 6-8 week follow-up for all the post-PRP injections, there was an average of $75 \%$ improvement in overall pain. At 6 months, 1 patient had no change, while 2 patients maintained the same reduction in pain at the 12- and 24-month follow-ups.

Limitations: This retrospective case series is only a small sample size of patients with refractory coccydynia.

Conclusions: Here we discuss the first case series of PRP for coccydynia. The results of the 3 patients in this case series are encouraging.

From : ${ }^{1}$ Progressive Spine and Orthopaedics Englewood, NJ; ${ }^{2} \mathrm{PMR} \&$ Interventional Pain Management, Kayal Orthopaedic Center, P.C., NJ and PMR at Northwell Health-Hofstra School of Medicine, NY

Author for correspondence: Fergie-Ross Montero-Cruz, DO

Address: Progressive Spine and Orthopedics, 440 Curry Ave., Suite A, Englewood, NJ 07631

E-mail: fmontero@progressivespine.net 
For a region which could be a cause of such tremendous, debilitating pain with limited treatment options, consideration for PRP into the coccyx and ligamentous support around it may have a significant role in refractory cases. What is encouraging about the case series is that 2 patients maintained response and pain improvement beyond 6 months and appear to have maintained the improvement to our follow-up at 12 months and 24 months. The patient with limited long-term improvement also has significant improvement with the PRP injection, though it did not last beyond 6 months. Although, it is important to note that the patient did experience pain improvement for 4 months longer than any other intervention the patient had undergone before.

Key words: coccydynia, coccyx pain, tailbone pain, platelet-rich plasma, platelet-rich plasma injection, PRP
Coccydynia is pain in the coccyx region. The most common cause of coccydynia is trauma, either from a direct axial force such as during a fall onto the coccyx or from cumulative trauma as a result of poor sitting mechanics (1). Risk factors for developing coccydynia include obesity, female gender and rapid weight loss (2). Due to its location, the coccyx is also susceptible to injury during difficult or instrumented childbirth. In addition, pain can arise from the pathology of the coccyx itself such as degeneration of the sacrococcygeal and intercoccygeal disc and joints, hypomobility or hypermobility of the sacrococcygeal joint, posterior subluxation of the coccyx, coccygeal retroversion or the presence of a bony spicule. Pain may also be referred from lumbar pathology or injury to the sacral nerve roots, spasm of the pelvic floor muscles or inflammation of the pericoccygeal soft tissues. Neoplasm, crystal deposition or infections are other possible causes of pain (2). The anatomy of the region is not well vascularized, and consists of the coccyx bones, and supporting ligaments and tendons (3).

Classically, patients present with localized pain over the coccyx that worsens with prolonged sitting or standing, leaning back while seated, and rising from a seated position (3). Physical examination reveals point tenderness over the coccyx with possible notable hypomobility or hypermobility of the sacrococcygeal joint (3). For a majority of patients, conservative management may be successful but for the remainder of patients, pain relief may only be transient and pain can become debilitating. Initial conservative management includes ergonomic adaptation, use of a cut-out cushion, pelvic therapy and manipulation therapy. For persistent pain, nonsurgical interventional procedures under fluoroscopic guidance are typically then trialed though there is yet to be widespread agreement on the best site of injection. Direct injections to the coccyx typically target the sacrococcygeal junction or the surrounding sacrococcygeal ligaments and consist of a local anesthetic with or without a steroid (3). Another approach is a caudal epidural steroid injection that primarily addresses sacral radicular pain. The ganglion impar, the pelvic segment of the sympathetic trunk, is also frequently targeted with the possibility of later proceeding with radiofrequency ablation (3). For patients with continued refractory pain the traditional next step and last resort is to seek surgical evaluation for a coccygectomy. A coccygectomy consists of a surgical amputation of the coccyx proximal to the sacrococcygeal junction (3). Current literature on coccygectomy for refractory coccydynia has shown success rates that range from $60-100 \%$ (4). The most frequent complication after coccygectomy is infection due to its close proximity to the rectum and anal canal with infection rates being report between $14.75 \%$ and $16.6 \%$ (1). Other complications include rectal injury, anal sphincter injury and incontinence $(1,4)$.

\section{BACKGROUND}

What has yet to be fully explored is the possible beneficial effect of local injection of platelet-rich plasma (PRP) for the treatment of refractory coccydynia. There are no published reports on coccydynia and PRP injection/treatment in current literature. PRP 
injections, however, have been successful in treating a variety of chronic tendon, ligament and bone injuries by inducing an inflammatory response to promote or re-initiate healing (4). PRP is autologous blood with a platelet concentration at least 4 times above normal baseline range (4). In addition to its role in clot formation, platelets also contain a variety of growth factors and cytokines that can stimulate the initial stage of the inflammatory process to incite the cascade of repair and healing. Additionally, PRP may also have an inhibitory effect on other cytokines that are thought to impede the early stages of healing. With this combined effect, PRP injection can reignite and accelerate the tissue healing process in non-healing or slowly healing injuries (5). Furthermore, PRP has been shown to have success with treating injury to tendon and ligaments which are chronic $(6,7)$. This is likely due to the limited vascular supply to these types of tissue. The coccyx region and supporting structures for it are analogous to this. Thus, we hypothesize that PRP will provide longer relief of coccydynia in patients who have failed traditional conservative and interventional techniques, such as local anesthetic and steroid injection.

\section{METHODS}

This retrospective case series with data collected through a retrospective chart review includes 3 patients with coccyx pain for greater than 6 months who presented to an outpatient pain management office in New York from 2014 until 2016, and failed prolonged relief with conventional treatments including physical therapy as well as local anesthetic and steroid injections. The patients underwent injection of the coccyx and ganglion impar in a series of 2 . The injections were done with fluoroscopic guidance, posterior to the coccyx, with field injections, and then the needle was driven to the anterior surface of the coccyx through the sacrococcygeal junction, for a ganglion impar block. The injections consisted of a mixture of $5 \mathrm{~mL}$ of $0.25 \%$ marcaine, and $40 \mathrm{mg}$ of triamcinolone. Each injection was performed with a 22-gauge 3.5-in spinal needle (8). Injection confirmation was done with radiopaque contrast for the ganglion impar block placement (Fig. 1). When the pain was deemed refractory for each patient, offering for PRP was done. This was presented with the option to repeat once again at the 6-8 week mark if needed.

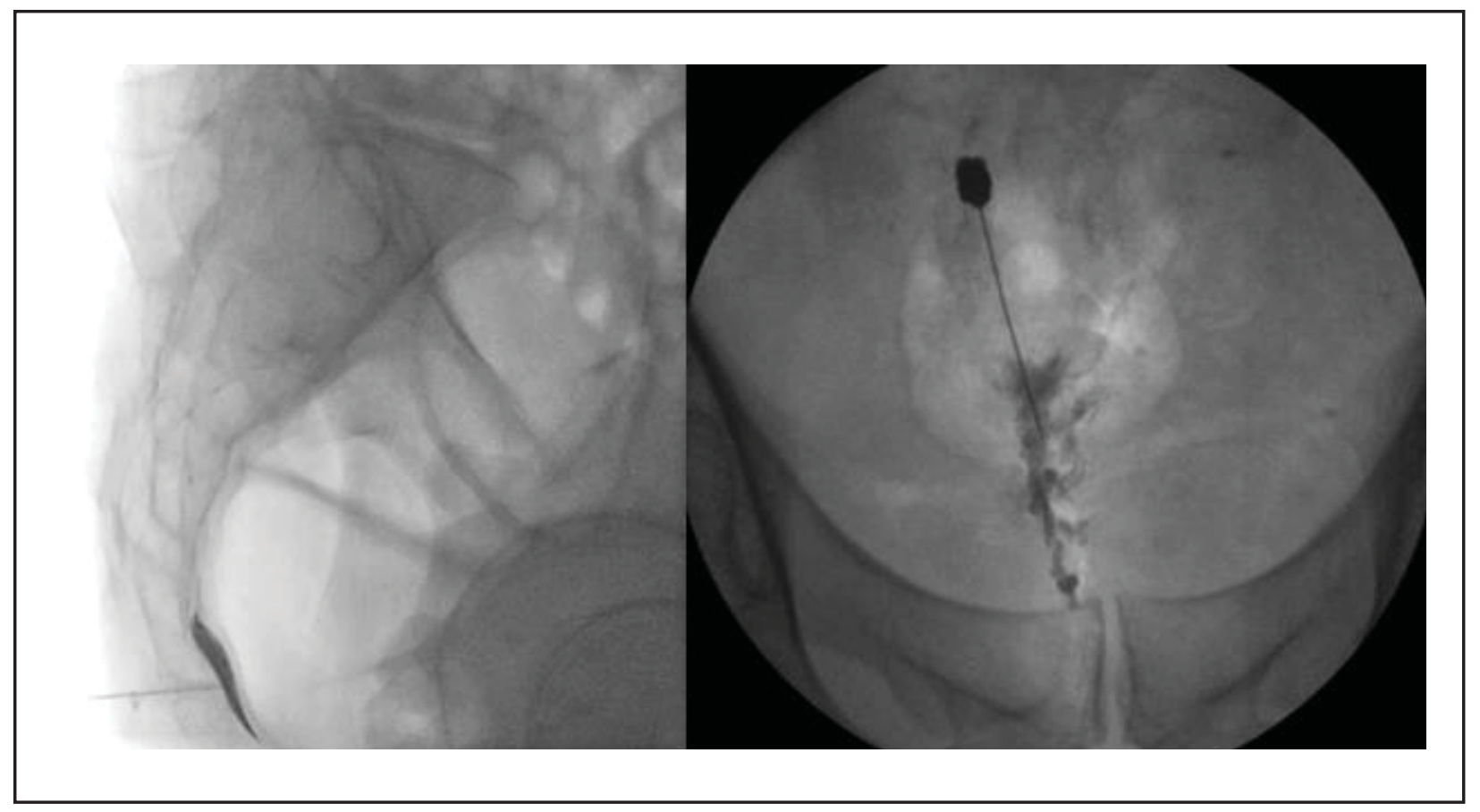

Fig. 1. Needle placement for coccyx and ganglion impar block in the lateral and anterior-posterior fluoroscopic views, with contrast spread for the ganglion impar block. 
All patients underwent PRP with fluoroscopic injection, and the use of the same PRP commercially available system; Magellan-Arteriocyte (9). The injection was done in the same fashion as the local anesthetic and steroid injection. The PRP injection was done along the posterior and anterior portions of the coccyx. Three $\mathrm{mL}$ of PRP were injected along the posterior aspect of the coccyx, and $3 \mathrm{~mL}$ of PRP in the anterior aspect of the coccyx. No contrast or local anesthetic was used in the injections, due to risk of it interrupting the PRP. The patients were evaluated with a numerical rating scale at $6-8$ weeks post-injection, 6 months post-injection, 12 months post-injection, and 24 months post-injection was recorded. Percentage of pain relief was then calculated. During the PRP injection, no NSAIDs, Aspirin or anti-inflammatory medications were used in the 10 days prior to the injection, and 6 weeks post-injection period.

\section{CASE DESCRIPTION}

We present 3 cases of refractory coccyx region pain who presented to an outpatient pain management office. All of which underwent conservative treatment and interventions. Each patient underwent PRP and was monitored over a 2-year period for prolonged relief. The patients were followed up at 6-8 weeks, considered for repeat, then seen again in 6-8 weeks, and then followed at 12 months and 24 months post injection. Each patient was treated with the MagellanArteriocyte PRP system. A summary of patient characteristics can be seen in Table 1.

The first case is a 50-year-old female who presented with 3 years of coccyx pain after a mechanical slip and fall on ice. Physical exam is significant for pain in the coccyx region, but otherwise is unremarkable. Standing and seated $x$-rays showed an anterior rotation of the coccyx from standing to sitting. No dislocation or subluxation was noted on x-ray imaging. An MRI showed no effusion, or any other process. The patient underwent traditional conservative treatment, including local anesthetic injections of Marcaine $0.25 \%$ with and without steroid, with only transient improvement of only 2 weeks and weeks improvement respectively. The patient then underwent a PRP injection along the posterior and anterior aspect of the coccyx. The patient had initial soreness for 2 weeks following the injection with symptom resolution noted at 8 weeks post-injection. The patient's pain scores on a numerical rating scale were 10 at time of injection, and 0 at the 8-week, 12-month and 24-month followups. The patient indicated a $100 \%$ improvement in pain following the injection.

The second case is a 64 year-old female with chronic tail bone pain following a slip and fall down stairs. She had presented with the symptoms for 1 year prior to presentation to our office. The pain was point tender over the distal tail bone, and was worse with sitting on firm surfaces. She underwent imaging with $x$-ray and MRI which showed no abnormalities of the sacrum and coccyx nor of the sacroiliac and pelvic regions. She had undergone physical therapy with modalities, as well as used a wedge cushion to help with the pain while sitting. Two ganglion impar blocks were performed resulting in a 50-60\% improvement in pain for 3 weeks. The patient then underwent PRP injection. Pre-injection pain score was 10 out of 10 on the numerical rating scale, which improved to $2-3$ out of 10 at 6 weeks following initial injection, amounting to about a $75 \%$ improvement in pain from original

Table 1. Patient summary.

\begin{tabular}{|l|l|l|}
\hline \multicolumn{1}{|c|}{ History } & \multicolumn{1}{|c|}{ Imaging Studies of Coccyx } & \multicolumn{1}{c|}{ Interventions } \\
\hline $\begin{array}{l}50 \text { yo F with coccydynia x 3 years s/p } \\
\text { mechanical slip and fall on ice }\end{array}$ & $\begin{array}{l}\text { XR - anterior rotation from standing } \\
\text { to seated } \\
\text { MRI - unremarkable }\end{array}$ & $\begin{array}{l}\text { - Physical therapy } \\
\text {-Donut cushion with moderate relief } \\
\text { - Coccyx and Ganglion Impar block x } 2\end{array}$ \\
\hline $\begin{array}{l}64 \text { yo F with coccydynia x 1 year s/p slip and } \\
\text { fall down stairs }\end{array}$ & $\begin{array}{l}\text { XR - unremarkable } \\
\text { MRI - unremarkable }\end{array}$ & $\begin{array}{l}\text { - Physical therapy } \\
\text { - Wedge cushion with minimal relief } \\
\text { - Coccyx and Ganglion Impar blocks x 2 }\end{array}$ \\
\hline 45 yo F with coccydynia x $>5$ years s/p MVA & $\begin{array}{l}\text { XR - unremarkable } \\
\text { MRI - slight effusion and edema in the } \\
\text { anterior portion of the coccyx }\end{array}$ & $\begin{array}{l}\text { - Physical therapy } \\
\text { - Manipulation therapy with intermittent relief } \\
\text { - Coccyx and Ganglion Impar blocks x 2 }\end{array}$ \\
\hline
\end{tabular}


baseline score. The PRP injection was repeated at the 8-week post-PRP injection mark when the preinjection pain score was once again 10 out of 10 . The patient received $50 \%$ improvement in pain after 6 weeks post-PRP injection and she rated her pain score a 5 out of 10 . This lasted for 6 months, before returning to baseline. The patient noted no change at 12 months, and 24 months.

The third case is a 45-year-old female with chronic tail bone pain following a motor vehicle accident. She presented to the office with a greater than 5-year history of pain in the region. On examination there was point tenderness along the distal tip of the coccyx. An x-ray showed no fracture or displacement, and the MRI showed a slight collection of fluid and an edema in the anterior portion of the coccyx. She had undergone physical therapy, manipulation therapy, as well as therapeutic and diagnostic injections of the coccyx and ganglion impar region. These resulted in pain reductions which were transient. She then underwent PRP injection and was followed up at the 8-week mark. Her pain prior to the injection was 9 out of 10 on the numerical rating scale, and at 8-week follow-up, she indicated a 2 out of 10 on the pain scale, which amounts to a $75 \%$ reduction in overall pain. The patient maintained this level of improvement in pain during 12-month and 24-month follow-ups.

\section{RESULTS}

Overall the 3 patients in this case series demonstrated significant improvement in numeric pain scale, as well as percentage of pain reduction as summarized in Table 2. At the 6-8-week follow-up for all the post-PRP injections, there was at least a $75 \%$ improvement in overall pain. At 6 months, 1 patient had no change, while 2 patients maintained the same reduction in pain at the 12- and 24-month follow-ups (Table 3).

\section{DISCUSSION}

Here we discuss the first case series of PRP for coccydynia. This is the first case series of its kind to be presented and published. No results on coccyx pain, coccydynia and PRP injection/treatment were found in the literature at the time of this paper submission. The results of the 3 patients in this case series are encouraging. Certainly, the sample size and design of this retrospective case series is limited and a larger sample size would be needed to improve generalizability. However, given the lack of literature support of PRP in other regions other than the lateral epicondyle, and other tendons, good data is not yet available on regions such as the coccyx. For a region which could be a cause of such tremendous, debilitating pain with limited treatment options, consideration for PRP into

Table 2. Post-injection results.

\begin{tabular}{|c|c|c|c|c|}
\hline Patient & $\begin{array}{c}\text { \% Improvement at } \\
\mathbf{6 - 8} \mathbf{~ w k}\end{array}$ & $\begin{array}{c}\text { \% Improvement at } \\
\mathbf{6 ~ m o}\end{array}$ & $\begin{array}{c}\text { \% Improvement at } \\
\mathbf{1 2} \text { mo }\end{array}$ & $\begin{array}{c}\text { \% Improvement at } \\
\text { 24 mo }\end{array}$ \\
\hline Patient 1 & $100 \%$ & $100 \%$ & $100 \%$ & $100 \%$ \\
\hline Patient 2 & $75 \%$ & N/A & N/A & N/A \\
\hline Patient 2 Repeat & $50 \%$ & $0 \%$ & $0 \%$ & $0 \%$ \\
\hline Patient 3 & $75 \%$ & $75 \%$ & $75 \%$ & $75 \%$ \\
\hline
\end{tabular}

Table 3. Pre-injection evaluation and summary.

\begin{tabular}{|c|c|c|c|c|c|}
\hline Patient & Injection Site & $\begin{array}{c}\text { Previous Surgery } \\
\text { Yes/No }\end{array}$ & $\begin{array}{c}\text { Pre-Injection Pain } \\
\text { Score (NRS 0-10) }\end{array}$ & Opioid & Procedure Done \\
\hline Patient 1 & Coccyx & No & 10 & No & PRP - Magellan \\
\hline Patient 2 & Coccyx & No & 10 & No & PRP - Magellan \\
\hline Patient 2 Repeat & Coccyx & No & 10 & No & PRP - Magellan \\
\hline Patient 3 & Coccyx & No & 9 & No & PRP - Magellan \\
\hline
\end{tabular}


the coccyx and ligamentous support around it may have a significant role in refractory cases. What is encouraging about the case series is that 2 of the patients have response and pain improvement that has remained beyond 6 months and appears to have maintained to follow-up at 12 months and even at 24 months. The patient with limited long-term improvement did have significant improvement with the PRP injection, though it did not last beyond 6 months. Although, it is important to note that this length of pain improvement was 4 months longer than any other interventions the patient had undergone before.

\section{CONCLUSION}

Conservative management may be successful in a majority of patients with coccydynia, but for the remainder of patients, coccydynia can become a chronic and debilitating injury. Interventional procedures using local anesthetic and steroid injection have shown to be of benefit for some patients, but the possible benefit from local injection of PRP for coccydynia has yet to be explored. Current research is generally supportive of the regenerative effects of PRP on chronic non-healing tendon injuries such as lateral epicondylitis or plantar fasciitis $(1,3)$. Unfortunately research on the effect of PRP on coccydynia is severely lacking and needs to be fully explored especially after having achieved promising results in the patients described $(1,10)$. Injection of PRP is believed to improve coccydynia by assisting in the repair and healing of the underlying tendon, ligament and/or bone injury to provide longer lasting pain relief. Given the outcomes in this limited case series, there is certainly a role for PRP injections which differ and offer another option for patients with refractory coccydynia aside from nerve blockage, caudal injection, or repeated steroid injections (7). In addition, local injections of PRP to the coccyx would be a less invasive option than surgery for patients who have exhausted all other treatment options and continue to struggle with the debilitating effects of coccydynia.

\section{REFERENCES}

1. Nathan S, Fisher B, Roberts C. Coccydynia. Bone \& Joint Journal 2010; 92:1622-1627.

2. Foye P. Coccydynia (coccygodynia). In L. Kunins (Ed.), UpToDate. Retrieved on May 6, 2017, from www.uptodate.com/contents/coccydynia-coccygodynia.

3. Lirette L, Chaiban G, Tolba R, Eissa H. Coccydynia: An overview of the anatomy, etiology, and treatment of coccyx pain. The Ochsner Journal 2014; 14:84-87.

4. Kwon HD, Schrot RJ, Kerr EE, Kim KD. Coccygodynia and Coccygectomy. Korean J Spine 2012;9:326-333.

5. Nguyen R, Borg-Stein J, Mclnnis K. Applications of platelet-rich plasma in musculoskeletal and sports medicine: An evidencebased approach. PM\&R 2011; 3:226-250.
6. Pavlovic V, Ciric M, Jovanovic V, Stojanovic P. Platelet rich plasma: A short overview of certain bioactive components. Open Medicine 2016; 11:242.

7. Sampson S, Gerhardt M, Mandelbaum B. Platelet rich plasma injection grafts for musculoskeletal injuries: A review. Current Rev MusculoMede 2008; 1: 165-174.

8. Datir A, Connell D. CT- guided injection for ganglion impar blockade. Clinical Radiology 2010; 65:21-25.

9. Arterioyte website. January 2016. Retrieved from www.arteriocyte.com/

10. Raeissadat SA, Sedighipour L, Rayegani SM, Bahrami MH, Bayat M, Rahimi R. Effect of PRP verse autologus blood on pain and function improvement in tennis elbow: A randomized clinical trial. Pain Res Treat 2014; 2014:191525. 